\title{
MURRAY ROSENBLATT'S CONTRIBUTIONS TO STRONG MIXING
}

\author{
By Richard C. Bradley
}

1. Introduction. Murray Rosenblatt's research has contributed much to the field of "strong mixing conditions," (i) by providing many results of his own in that field, and (ii) by inspiring a vast amount of research in that field by other people. This note will give just a small snapshot of (i) and an even smaller snapshot of (ii).

Within the broader field of probability theory, the field of "strong mixing conditions" seemed to "take off" with the publication in 1956 of the paper of Rosenblatt [17] titled "A central limit theorem and a strong mixing condition." Before that, weaker notions of "mixing" had been studied by Eberhard Hopf [9] and others in ergodic theory; and "strong" forms of "mixing" had been implicitly present in the work of Doeblin [4] involving what is now known as "Doeblin's condition" for Markov chains, and also in Doeblin's [5] work on continued fractions (see Iosifescu [13] for details on this latter work). But it was Rosenblatt's [17] paper — which introduced the "strong mixing" (" $\alpha$-mixing") condition, showed how it "coordinated" well with a Bernstein-type blocking scheme and produced a central limit theorem under that condition — that really seemed to light a spark and lead to the development of "strong mixing conditions" as a field of its own. In particular, the 1956 paper inspired a considerable number of papers by other researchers in which variants of "strong mixing conditions" were proposed and used in various types of limit theorems. Those papers include, among many others, Ibragimov [10, 11], Kolmogorov and Rozanov [14], and Cogburn [2]. Subsequently, the field of "strong mixing conditions" exploded into an active and vibrant area of research.

Strong mixing conditions and the associated central limit theorems have enjoyed broad appeal beyond probability and into the mainstream statistics community. Applications of strong mixing include applications as diverse as block or stationary bootstrapping of time series (see e.g., Politis and Romano [16]); inference for linear time series; and parametric and nonparametric inference for nonlinear time series (see e.g., Fan and Yao [7]). Strong mixing is often the key tool for establishing asymptotic normality of various estimators of complicated time series models. In particular, inference for popular financial time series models such as GARCH and stochastic volatility models, often rely on the process being strongly mixing.

A short note such as this on Rosenblatt's work on strong mixing conditions cannot do justice to all of his own contributions to this field, and it cannot even begin to touch on the many key advances in this field that have been made by others. For details on the 
history of the development of the field, see e.g. [6] or the series [1]. This article will provide a sampling of just a few of the many contributions that Rosenblatt has made to this field, with a couple of examples of their direct inspiration of subsequent research by others.

To set the table for the discussion to follow, the rest of Section 1 here will be devoted to the definition of strong mixing and two other mixing conditions.

Suppose $(\Omega, \mathcal{F}, P)$ is a probability space. For any two $\sigma$-fields $\mathcal{A}$ and $\mathcal{B} \subset \mathcal{F}$, define the following three measures of dependence:

$$
\begin{aligned}
\alpha(\mathcal{A}, \mathcal{B}) & :=\sup _{A \in \mathcal{A}, B \in \mathcal{B}}|P(A \cap B)-P(A) P(B)|, \\
\phi(\mathcal{A}, \mathcal{B}) & :=\sup _{A \in \mathcal{A}, B \in \mathcal{B}, P(A)>0}|P(B \mid A)-P(B)|, \quad \text { and } \\
\rho(\mathcal{A}, \mathcal{B}) & :=\sup |\operatorname{Corr}(f, g)|
\end{aligned}
$$

where this last supremum is taken over all pairs of square-integrable random variables $f$ and $g$ such that $f$ is $\mathcal{A}$-measurable and $g$ is $\mathcal{B}$-measurable.

Now suppose $X:=\left(X_{k}, k \in \mathbf{Z}\right)$ is a strictly stationary sequence of random variables on our given probability space $(\Omega, \mathcal{F}, P)$. For each positive integer $n$, define the following three dependence coefficients:

$$
\begin{aligned}
& \alpha(n):=\alpha\left(\sigma\left(X_{k}, k \leq 0\right), \sigma\left(X_{k}, k \geq n\right)\right), \\
& \phi(n):=\phi\left(\sigma\left(X_{k}, k \leq 0\right), \sigma\left(X_{k}, k \geq n\right)\right), \quad \text { and } \\
& \rho(n):=\rho\left(\sigma\left(X_{k}, k \leq 0\right), \sigma\left(X_{k}, k \geq n\right)\right) .
\end{aligned}
$$

Here and below, the notation $\sigma(\ldots)$ means the $\sigma$-field of events generated by (..). The (strictly stationary) sequence $X$ is said to be "strongly mixing" (or " $\alpha$-mixing") if $\alpha(n) \rightarrow 0$ as $n \rightarrow \infty$, " $\phi$-mixing" if $\phi(n) \rightarrow 0$ as $n \rightarrow \infty$, and " $\rho$-mixing" if $\rho(n) \rightarrow 0$ as $n \rightarrow \infty$. The "strong mixing" ( " $\alpha$-mixing") condition is the one that was introduced and used in a central limit theorem in the 1956 paper of Rosenblatt [17]. The " $\phi$-mixing" condition was introduced by Ibragimov [10] and was studied by both Cogburn [2] and Ibragimov [11]. The " $\rho$-mixing" condition was introduced by Kolmogorov and Rozanov [14]. (The "maximal correlation coefficient" defined in (1.3) had been studied earlier in statistical contexts that did not involve "random sequences.") It is well known that $\phi$-mixing implies $\rho$-mixing, that $\rho$-mixing implies strong mixing ( $\alpha$-mixing), and that the converses of both of those statements are false. For a formulation of other classic strong mixing conditions and their connections with the ones here, see e.g. [6] or [1, V1, Chapters 3 and 5].

2. Markov chains. An ongoing theme of research by many people is the question of what strong mixing properties are satisfied by random sequences with various other specific types of dependence structure. The books by Rosenblatt [20, 24, 26] gave (along with much other material) a treatment of different facets of this general question. In particular, 
strong mixing conditions for strictly stationary Markov chains (with, say, state space $\mathbf{R}$ ) has been a topic of much research by Rosenblatt. A small selection of specific contributions of his on this topic will be briefly reviewed here.

Strong mixing conditions for Markov chains are treated extensively in Chapter 7 of Rosenblatt's 1971 book [20] on Markov processes. Along with much other information, that chapter gave what were, to the best of my knowledge, (1) the first known examples of strictly stationary Markov chains that are $\phi$-mixing in the "usual" direction of time but fail to be $\phi$-mixing in the "reversed" direction of time, and (2) the first known examples of strictly stationary $\rho$-mixing Markov chains such that for every positive integer $n$, the $n$-step (conditional) transition probability distributions are almost surely singular with respect to the marginal distribution.

Central limit theory under the $\rho$-mixing condition apparently started with central limit theorems of Rosenblatt [19] [20, Chapter 7] for square-integrable "instantaneous" functions of strictly stationary $\rho$-mixing Markov chains, and also with his paper [18] giving central limit theorems for some kernel-type estimators of marginal probability density for $\rho$-mixing Markov chains with an absolutely continuous marginal distribution. Shortly thereafter, the development of central limit theory for general (not necessarily Markovian) strictly stationary $\rho$-mixing sequences was promoted by Ibragimov [12]. (For more on that theory, see e.g. [1, V1, Chapter 11].)

In [18] and [20, Chapter 7], Rosenblatt used the Riesz Convexity (Interpolation) Theorem to prove the equivalence of a class of mixing conditions (including $\rho$-mixing) for Markov chains. That was apparently the first use of "interpolation theorems" of that type for comparison of different mixing conditions. This work subsequently inspired a more extensive use (not restricted to Markov chains) of various interpolation theorems to establish "covariance" and "cumulant" inequalities under various strong mixing conditions, and to compare different measures of dependence on which strong mixing conditions are based. For some of the main results and history of that later development, see [1, V1, Chapter 4], including the notes at the end of that chapter.

In [23], Rosenblatt showed that (in essence) an ultra-strong version of a "Markov regularity condition" that had been used in various papers, could not be satisfied by strictly stationary Markov chains other than the i.i.d. sequences. Around the same time, Rosenblatt conjectured to me that this condition could not be satisfied by any strictly stationary sequences, Markovian or not, except the i.i.d. sequences. Shortly thereafter, I was able to confirm the conjecture. For a detailed exposition, see [1, V2, Theorem 24.2].

3. A little known contribution. A remarkable paper of Rosenblatt [21] contains some ingenious fundamental insights in connection with the strong mixing condition for Markov chains. Unfortunately, this paper seems to have been practically unknown for at least thirty years after its publication. In this section, the content and the connections of this paper with the work of other researchers will be reviewed in some detail. 
It will be useful to first formulate a classic result of Doeblin [4] (just the "aperiodic" case) involving what is now known as "Doeblin's condition" for Markov chains. It is well known that Doeblin's result can be reformulated in a different but equivalent way in terms of the $\phi$-mixing condition (which was defined a couple of decades later), as follows:

Theorem 3.1 (Doeblin (reformulated)). Suppose $X:=\left(X_{k}, k \in \mathbf{Z}\right)$ is a strictly stationary Markov chain which is ergodic, aperiodic, and satisfies $\phi(n)<1$ for some $n \geq 1$. Then $\phi(n) \rightarrow 0$ (at least exponentially fast) as $n \rightarrow \infty$.

We shall return to this theorem below, after picking up some other threads.

For strictly stationary random sequences, Cogburn [2] introduced, under the name "uniform ergodicity," a "Cesaro" variant of the strong mixing condition, and (along with other results) proved a central limit theorem involving this condition. The uniform ergodicity condition need not be formally defined here. Now (for strictly stationary sequences) strong mixing implies uniform ergodicity, and uniform ergodicity implies ergodicity. A natural question, pertinent to the development of limit theory for strictly stationary sequences, is how much weaker uniform ergodicity is than strong mixing. Rosenblatt [21] examined this question and proved the following result involving Markov chains:

Theorem 3.2 (Rosenblatt). Suppose $X:=\left(X_{k}, k \in \mathbf{Z}\right)$ is a strictly stationary Markov chain which is "mixing" (in the ergodic-theoretic sense) and satisfies uniform ergodicity; then $\alpha(n) \rightarrow 0$ as $n \rightarrow \infty$ (strong mixing).

In the same paper, Rosenblatt [21] also suggested that this result might still hold for general strictly stationary sequences (i.e., without the Markov assumption). That now seems to be an open question, with (so far) no decisive evidence either way.

Formally, Theorem 3.2 was the "goal" of Rosenblatt's [21] paper. However, the various arguments in this paper contained further peculiar and ingenious insights that have remained little known. In particular, Rosenblatt showed that if $X:=\left(X_{k}, k \in \mathbf{Z}\right)$ is a strictly stationary Markov chain that fails to satisfy strong mixing, then there exists a number $q \in(0,1)$ such that the following condition holds: For every positive integer $N$ and every $\theta>0$, there exist events $G \in \sigma\left(X_{k}, k \leq 0\right)$ and $H \in \sigma\left(X_{k}, k \geq N\right)$ such that $q-\theta<P(H)<q+\theta$ and $P(G \triangle H)<\theta$. (Here $\triangle$ denotes symmetric difference. Obviously, adjusting the parameters a little, one can have $q-\theta<P(G)<q+\theta$ as well.) Rosenblatt also showed that this condition holds for every $q \in(0,1)$ under the additional assumption of a trivial (past or) future "tail $\sigma$-field." Combining this fact with $q=1 / 2$ and other arguments in Rosenblatt's [21] paper, implicitly yields the following theorem:

Theorem 3.3 (Rosenblatt). Suppose $X:=\left(X_{k}, k \in \mathbf{Z}\right)$ is a strictly stationary Markov chain which is ergodic, aperiodic, and satisfies $\alpha(n)<1 / 4$ for some $n \geq 1$. Then $\alpha(n) \rightarrow 0$ as $n \rightarrow \infty$. 
Now for any two $\sigma$-fields $\mathcal{A}$ and $\mathcal{B}$, one has by trivial arguments that $\alpha(\mathcal{A}, \mathcal{B}) \leq 1 / 4$ and $\phi(\mathcal{A}, \mathcal{B}) \leq 1$. Hence for a given strictly stationary sequence (Markovian or not), one has that $\alpha(n) \leq 1 / 4$ and $\phi(n) \leq 1$ for every positive integer $n$. Thus Theorem 3.3 is a nearly exact analog, for strong mixing, of Doeblin's result (transcribed to " $\phi$-mixing") in Theorem 3.1. The only significant difference is that in the context of Theorem 3.1, the mixing rate has to be (at least as fast as) exponential, whereas in Theorem 3.3, the mixing rate need not be exponential (as was shown, for example, for the Markov chains studied by Davydov [3]). Yet while Theorem 3.1 receives frequent attention in the literature, Theorem 3.3 was apparently "absent from the radar screen" for three decades after the publication of $[21]$.

For a detailed exposition of the material in Rosenblatt's [21] paper (including Theorems 3.2 and 3.3 and the other insights from that paper described here), see [1, V2, Sections 21.25-21.28 and 24.17-24.22].

4. Strong mixing conditions and estimation problems for random fields. At this point, we depart from Markov chains and turn to some contributions of Rosenblatt involving more general random sequences as well as random fields.

As Richard Olshen explained to me about 30 years ago, a major motivation behind Rosenblatt's [17] paper and the subsequent development of the field of strong mixing conditions, was to provide a way of proving limit theorems for use in statistical inference for data from time series that seemed to be "weakly dependent" but did not necessarily seem to "fit" more specific models of dependence such as Markov chains, martingales, Gaussian sequences, or ARMA models. Since then, in that spirit, a vast amount of research has been done by many researchers on statistical estimation of various kinds under strong mixing conditions. The 1985 book of Rosenblatt [24] was a prominent treatise on this topic. Further, much of that book gave a treatment of random fields (not just random sequences) satisfying strong mixing conditions, an ongoing topic of research of Rosenblatt and many others. We shall return to that book after providing some background information.

Suppose $d$ is a positive integer, and $X:=\left(X_{k}, k \in \mathbf{Z}^{d}\right)$ is a strictly stationary random field. For a given positive integer $n$, define

$$
\alpha^{*}(n):=\sup \alpha\left(\sigma\left(X_{k}, k \in A\right), \sigma\left(X_{k}, k \in B\right)\right),
$$

where the supremum is taken over all pairs of nonempty, disjoint sets $A, B \subset \mathbf{Z}^{d}$ such that

$$
\operatorname{dist}(A, B):=\min _{a \in A, b \in B}\|a-b\| \geq n .
$$

Here, for a given $k \in \mathbf{Z}^{d}$, $\|k\|$ denotes the Euclidean norm of $k$. The (strictly stationary) random field $X$ will be said to be " $\alpha^{*}$-mixing" if $\alpha^{*}(n) \rightarrow 0$ as $n \rightarrow \infty$.

In the case $d=1$ (the classic case of strictly stationary random sequences), $\alpha^{*}$-mixing is a stronger condition than the strong mixing ( $\alpha$-mixing) condition (involving (1.4)), because in (4.1) the two index sets $A$ and $B$ are not restricted to "past" and "future" (as 
in (1.4)); they can instead be "interlaced," with each set having elements between ones in the other set.

Extending well known arguments and results of Kolmogorov and Rozanov [14] involving the usual strong mixing and $\rho$-mixing conditions in the case of stationary Gaussian sequences, Rosenblatt [22] showed that for a given stationary Gaussian random field $X:=\left(X_{k}, k \in \mathbf{Z}^{d}\right)$, (i) the $\alpha^{*}$-mixing condition is equivalent to the corresponding " $\rho^{*}$ mixing" condition (defined using in the right hand side of (4.1) the maximal correlation coefficient from (1.3)), and (ii) those equivalent conditions are satisfied if $X$ has a continuous positive spectral density function. (An exposition of those results was also given in $[24$, pp. 73-77].)

Later, it became known that observation (i) holds for general (not necessarily Gaussian) strictly stationary random fields $X:=\left(X_{k}, k \in \mathbf{Z}^{d}\right)$. (See [1, V3, Theorem 29.12(II)(A)] for the result, its proof, and its history.) We shall continue to use the term " $\alpha$-mixing" (instead of " $\rho^{*}$-mixing") in the discussion below. Of course in contrast, for random sequences (the case $d=1$ ), the strong mixing ( $\alpha$-mixing) condition (based on (1.4), with the two index sets being just "past" and "future") is strictly weaker than the $\rho$-mixing condition (based similarly on (1.6)).

(In a slightly different vein, the paper of Rosenblatt [22] also gave a central limit theorem for random fields under "martingale-like" assumptions similar to those used by Gordin [8] in a classic central limit theorem for random sequences.)

Now let us return to the 1985 book of Rosenblatt [24]. This book gives an extensive treatment of various estimation problems for strictly stationary $\alpha$-mixing random sequences $X:=\left(X_{k}, k \in \mathbf{Z}\right)$ and strictly stationary $\alpha^{*}$-mixing random fields $X:=\left(X_{k}, k \in\right.$ $\mathbf{Z}^{d}$ ). The problems considered there include the estimation of (under appropriate assumptions) autocovariances, spectral densities, higher-order spectral densities, marginal probability densities, and regression functions. The book contains a wealth of results and opened up a broad spectrum of new research problems.

In the formulation of most of the theorems in the 1985 book involving $\alpha$-mixing or $\alpha^{*}$-mixing, there is no assumption on the rate of convergence of $\alpha(n)$ or $\alpha^{*}(n)$ to 0 ; instead the "mixing rate" is allowed to be arbitrarily slow. To compensate, there typically are assumptions on higher-order moments and/or higher-order cumulants. For example, in a central limit theorem for some estimators of spectral density for strictly stationary random fields, Rosenblatt [24, p. 157, Theorem 7] assumes (in addition to $\alpha^{*}$-mixing) the summability of cumulant functions of the $X_{k}$ 's up to order 8 . Now the usual estimators of spectral density (such as ones based on periodograms) involve quadratic forms of the $X_{k}$ 's, and hence the natural basic moment assumption for a central limit theorem for those estimators is $E X_{0}^{4}<\infty$, in order for the quadratic forms to have finite second moments. A couple of years after the publication of his book, Rosenblatt [25] posed the problem of whether in theorems such as the one alluded to here, one could get by with 
just the summability of cumulants of order 4 (instead of order 8), or even with just the assumption of finite fourth moments (of course in addition to the assumption of $\alpha^{*}$-mixing).

In his 1994 Ph.D. thesis at Indiana University (see also [15, Theorem 2]), Curtis Miller gave an affirmative answer to that question for a class of estimators that involve "blocks" of the $X_{k}$ 's. Miller's result only assumes $E X_{0}^{4}<\infty$; no assumption of moments of higher than fourth order and no further assumption on covariances or cumulants are required. (Miller formally used the " $\rho^{*}$-mixing" condition, but under strict stationarity that condition is equivalent to $\alpha^{*}$-mixing as noted above.) The question is apparently still open for other estimators of spectral density, such as ones that involve averaging the periodograms over neighboring frequencies. Anyhow, this is just another particular example of the many ways in which the work of Rosenblatt on strong mixing conditions has directly inspired the research in the field by others, including young researchers.

Starting with his 1956 paper [17], Murray Rosenblatt has contributed a great deal to the field of strong mixing conditions, and has helped establish it as an active field of research. His work has directly inspired many contributions in that field by other researchers. Strong mixing conditions continue to be an active field of research that has important modeling and inference ramifications in a variety of applications.

Acknowledgement: My thanks go to Richard Davis, who made many helpful comments and suggestions and provided the third paragraph of this article.

\section{References}

[1] R.C. Bradley. Introduction to Strong Mixing Conditions, Volumes 1, 2, and 3. Kendrick Press, Heber City (Utah), 2007.

[2] R. Cogburn. Asymptotic properties of stationary sequences. Univ. Calif. Publ. Statist. 3 (1960) 99-146.

[3] Yu.A. Davydov. Mixing conditions for Markov chains. Theor. Probab. Appl. 18 (1973) 312-328.

[4] W. Doeblin. Sur les propriétés asymptotiques de mouvement régis par certains types de chaines simples. Bull. Math. Soc. Roum. Sci. 39 (1937), no. 1, 57-115, no. 2, 3-61.

[5] W. Doeblin. Remarques sur las théorie métriques des fractions continues. Compositio Math. 7 (1940) 353-371.

[6] P. Doukhan. Mixing: Properties and Examples. Springer, New York, 1995.

[7] J. Fan and Q. Yao. Nonlinear Time Series: Nonparametric and Parametric Methods. Springer, New York, 2003.

[8] M.I. Gordin. The central limit theorem for stationary processes. Soviet Math. Doklady 10 (1969) 1174-1176. 
[9] E. Hopf. Ergodentheorie. Springer-Verlag, Berlin, 1937.

[10] I.A. Ibragimov. Some limit theorems for stochastic processes stationary in the strict sense. Dokl. Akad. Nauk. SSSR 125 (1959) 711-714.

[11] I.A. Ibragimov. Some limit theorems for stationary processes. Theor. Probab. Appl. 7 (1962) 349-382.

[12] I.A. Ibragimov. A note on the central limit theorem for dependent random variables. Theor. Probab. Appl. 20 (1975) 135-141.

[13] M. Iosifescu. Doeblin and the metric theory of continued fractions: A functional theoretic solution to Gauss' 1812 problem. In: Doeblin and Modern Probability, (H. Cohn, ed.), pp. 97-110. Contemporary Mathematics 149. American Mathematical Society, Providence (Rhode Island), 1993.

[14] A.N. Kolmogorov and Yu. A. Rozanov. On strong mixing conditions for stationary Gaussian processes. Theor. Probab. Appl. 5 (1960) 204-208.

[15] C. Miller. A CLT for the periodograms of a $\rho^{*}$-mixing random field. Stochastic Process. Appl. 60 (1995) 313-330.

[16] D.N. Politis and J.P. Romano. The stationary bootstrap. J. Amer. Statist. Assoc. 89 (1994) 1303-1313.

[17] M. Rosenblatt. A central limit theorem and a strong mixing condition. Proc. Natl. Acad. Sci. USA 42 (1956) 43-47.

[18] M. Rosenblatt. Density estimates and Markov sequences. In: Nonparametric Techniques in Statistical Inference, (M.L. Puri, ed.), pp. 199-210. Cambridge University Press, London, 1970.

[19] M. Rosenblatt. A prediction problem and central limit theorems for stationary Markov sequences. In: Proceedings of the Twelfth Biennial Seminar of the Canadian Mathematical Congress on Time Series and Stochastic Processes; Convexity and Combinatorics (Vancouver, B.C., 1969), (R. Pyke, ed.), pp. 99-114. Canadian Mathematical Congress, Montreal, 1970.

[20] M. Rosenblatt. Markov Processes. Structure and Asymptotic Behavior. SpringerVerlag, Berlin, 1971.

[21] M. Rosenblatt. Uniform ergodicity and strong mixing. Z. Wahrsch. verw. Gebiete 24 (1972) 79-84.

[22] M. Rosenblatt. Central limit theorems for stationary processes. Proceedings of the Sixth Berkeley Symposium on Probability and Statistics, Volume 2, pp. 551-561. University of California Press, Los Angeles, 1972.

[23] M. Rosenblatt. Some remarks on a mixing condition. Ann. Probab. 7 (1979) 170-172.

[24] M. Rosenblatt. Stationary Sequences and Random Fields. Birkhäuser, Boston, 1985.

[25] M. Rosenblatt. Private communication, 1987.

[26] M. Rosenblatt. Gaussian and Non-Gaussian Linear Time Series and Random Fields. Springer, New York, 2000. 